\title{
Reverse Trendelenburg
}

National Cancer Institute

\section{Source}

National Cancer Institute. Reverse Trendelenburg. NCI Thesaurus. Code C62169.

A supine position with the person inclined at an angle of 45 degrees so that the head is higher than the pelvis. 